\title{
A design of stretcher with auxiliary functions of lateral positioning and transferring for immobilized patients
}

\author{
Ying Xiong ${ }^{1}$, Xuehua Tang $^{2}$, Congcong Shi ${ }^{1, *}$, and Yang Yang ${ }^{1}$ \\ ${ }^{1}$ Mechanics Institute, Shanghai dianji university, Shanghai 201306 P. R. China \\ ${ }^{2}$ School of design and art, Shanghai dianji university, Shanghai 201306 P. R. China
}

\begin{abstract}
For now, many hospitals that require nurses to move patients by hand from stretchers to a hospital bed, so a design of stretcher with auxiliary functions of lateral positioning and transferring for immobilized patients, which is a mechanical mechanism consisted of rigid rods, joints and sliders, was designed to help the nurses to move patients between beds and reduce their workload. Driven by motors, the rigid rods can be rotated, stretched or shortened so that the entire stretcher bed board can archive to a proper posture and position. In this paper, the following objectives will be achieved: (i) Create a schematic of the mechanism and describe the principles and functions (ii) the calculation of inverse kinematics, forward kinematics, dynamics (including energy), and PD control in the mechanism (iii) The motion process of simulating the mechanism using MATLAB (iv) Using MATLAB to create the plots of angle, torque, and position state (v) Using SolidWorks to construct the prototype and to implement the motion simulation of the mechanism (vi) Describe the practical application and future Extensions of this mechanism.
\end{abstract}

\section{Introduction}

Every year, there are thousands of patients around the world. Some of them need to lie on a bed for temporary or permanent due to loss of mobility for various reasons, such as a fracture, an anesthesia, a stroke, a mole, an amputation or other illness. When the patient has undergone surgery, they need to be transferred from the operating table to the stretcher and then transferred from the stretcher to the hospital bed. There is a problem that has not been effectively solved for a long time, which is to transfer the immobilized patients smoothly and easily between the operating table and the stretcher, between the stretcher and the hospital bed, or between two hospital beds. Until now, in most parts of the world, moving patients between beds requires nurses to carry them by hand. In China, because of the shortage of nurses, the family members of the patients are even needed to transfer the immobilized patients. This is a common phenomenon ([1], [2]). However, on the one hand, this is a matter that needs to be taken seriously. Because moving patients is not a simple matter, any inappropriate operation may hurt them and make them feel uncomfortable ;

\footnotetext{
*Corresponding author: smileccong@,foxmail.com
} 
more seriously, that may aggravate their wounds. On the other hand, this is a heavy burden for the nurse. Nurses are mostly women, they don't have much strength, and if patients are heavier, they have a hard time moving patients. In addition, if some patients are very heavy, they will be more difficult to complete the task. If the family members try to help move the patient, they will be more difficult to do this because they have not received professional training ([3]). If there are some mistakes, that will increase the workload of the medical staff. Therefore, it is very necessary to design some mechanisms to help them to achieve this task more easily and safely, while reducing the burden on the nurses and patients' families, when transfer the immobilized patients between beds.

\section{Research status}

Many medical personnels, professors, doctors and other researchers are aware of this problem, and they are trying to solve it or modify it. Moreover, they have put forward novel ideas, published many articles, applied for many patents, and developed a lot of products ([4]). Each of these designs has its own properties, and most have proven to be effective. Although these designs have their own advantages, they also have some disadvantages. For example, there is a university laboratory that designs a mechanism similar to a crane in a factory, including a load-bearing unit, a lifting unit, and a bracket unit. The load-bearing unit includes two vertically-symmetrical support standers that are fixedly disposed and fixedly connected to the top end thereof; a horizontal crossbar; the lifting unit comprises a lifting group and a translation group, and the lifting group comprises two fixed pulleys respectively connected to the two ends of the horizontally arranged lifting rail through the lifting rope, the fixed pulley is fixed on the horizontal crossbar; the translation group is the translation pulley slides along the lifting rail; the lower end of the translation pulley is connected to the horizontally arranged bracket unit. When using it, the immobilized patient can be fixed to the load-bearing unit, then the patient is lifted by the lifting unit, then translated to another bed, and then the patient is slowly placed on the bed board. The biggest advantage of this mechanism is that it saves manpower, so the nurse only needs to fix the patient and release the patient by manipulating the motor. But its biggest disadvantage is that the bracket is too large and the bracket itself is not easy to move ([5]). In addition, there is a hospital researcher who came up with an idea to suggest a bridge-like mechanism between the two beds. It includes a base for straddle the two beds, and a plurality of freely rotatable pulleys are arranged at the top of the base, each of which has a top surface that is higher than the top surface of the base. When the patient is to be transferred, simply lift the sheets and place the patient's center of gravity, that is, the buttocks, on the pulley to facilitate the transfer of the patient between the beds. The biggest advantage of this structure is that it does not require the use of motors and other drives, and it is simple in construction and no need extra motor. However, its biggest drawback is poor comfort, because many patients are vulnerable and cannot be transferred in this way ([6]). A company's researchers have developed a patient lifting transfer device for use in the medical field, which is made of high-strength polycarbonate transparent sheet, with a micro-rolling bearing between the small slide and the main transfer plate that slides relatively easily. The present invention can be easily inserted under the patient's body to conveniently move the patient between the hospital bed and the cart and the operating table. The advantage of this mechanism is that it is easy to install and use, but the comfort is not good enough ([7]). In addition, a medical school has given a solution that is designed to eliminate the pain due to external causes when the patient is transferred to a hospital bed. The key component is a pair of stretcher rods and a centrally detachable soft sheet composed of the stretcher bed and the cooperation or assistance of the hand-pushing bed and the hospital bed are finally completed by the three parties: during the whole process of 
transferring the immobilized patient under the nurse's assistant, the patient's own physical posture is always keep constant, that is, it does not cause the patient to increase the painful feeling due to the formation of the process of transferring. The advantage of this solution is that it has low production cost and easy to use, but it only relieves the suffering of the patient and does not effectively relieve the burden on the nurse, who still needs to move the patient by hand ([8]). Therefore, the team intend to design a simple and easy-to-operate mechanism to help nurses transfer immobilized patients between different beds, for reducing their burden and avoiding secondary harm to patients.

\section{Design}

\subsection{Mechanism specification}

For the mechanism of the Stretcher with Auxiliary Functions of Lateral Positioning and Transferring for Immobilized Patients, the free body diagram was shown in Fig. 1 below. There are four joints: Joint A and Joint B are fixed on the ground, and Joint C and Joint D are linked by rigid rod $\mathrm{CD}$. All of the four joints can be rotated. Also, there are two sliders: Slider AC and Slider BD, which can be stretched or shortened by motors so that the entire stretcher bed board can archive to a proper posture and position. Thus, there are total three degree of freedom in this mechanism.

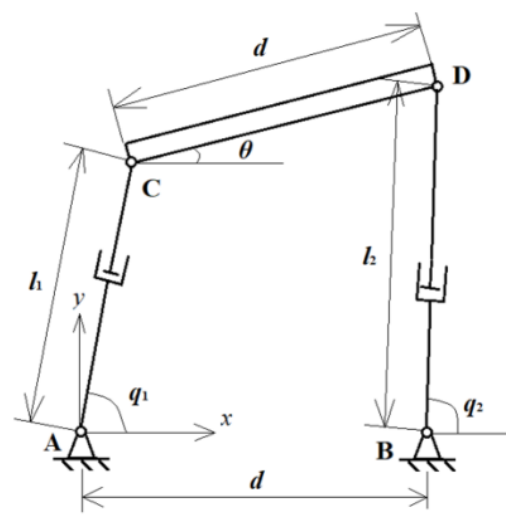

Fig. 1. Free body diagram.

The symbols in Fig. 1 was explained on the Table 1 shown below.

Table 1. Symbols and notations.

\begin{tabular}{|c|l|}
\hline SYMBOLS & NOTATIONS \\
\hline 11 & Length of Slider AC \\
\hline 12 & Length of Slider BD \\
\hline $\mathrm{d}$ & Length of Rigid Rod CD \\
\hline $\mathrm{q} 1$ & Angle betweern Slider AC and horizontal \\
\hline $\mathrm{q} 2$ & Angle betweern Slider BD and horizontal \\
\hline$\theta$ & Angle betweern Rod CD and horizontal \\
\hline
\end{tabular}

In original status, both Slider AC and Slider BD perpendicular to the horizontal plane and they have same length. But the distance between Joint A and Joint B, and length of Rigid Rod CD are always identical which is d. 


\subsection{Inverse kinematics}

Use the position of Joint $\mathrm{C}$ and angle $\theta$ to describe the state of the stretcher. Three actuators are respectively installed on $\mathrm{q}_{1}, \mathrm{q}_{2}$ and $\mathrm{l}_{1}$. Joint $\mathrm{A}$ is at the origin of the coordinate. To implement the inverse kinematics, $\mathrm{x}_{\mathrm{C}}, \mathrm{y}_{\mathrm{C}}$ and $\theta$ are known. Thus, $\mathrm{q}_{1}, \mathrm{q}_{2}$, $\mathrm{l}_{1}$ and $\mathrm{l}_{2}$ need to be solved.

$$
\begin{gathered}
l_{1}=\sqrt{x_{C}{ }^{2}+y_{C}^{2}} \\
q_{1}=\operatorname{atan2}\left(y_{C}, x_{C}\right) \\
x_{D}=x_{C}+d C_{\theta}, y_{D}=y_{C}+d S_{\theta} \\
l_{2}=\sqrt{\left(x_{D}-d\right)^{2}+y_{D}^{2}}=\sqrt{\left(x_{C}+d C_{\theta}-d\right)^{2}+\left(y_{C}+d S_{\theta}\right)^{2}} \\
q_{2}=\operatorname{atan} 2\left(y_{D}, x_{D}-d\right)=\operatorname{atan} 2\left(y_{C}+d S_{\theta}, x_{C}+d C_{\theta}-\mathrm{d}\right)
\end{gathered}
$$

Therefore, there always have solutions for inverse kinematics as long as $l_{1}$ and $l_{2}$ extend long enough. This RPR joint give the mechanism wide workspace.

\subsection{Forward kinematics}

Choose actuators at $\mathrm{q}_{1}, \mathrm{l}_{1}$ and $\mathrm{l}_{2}$. As the motors can be installed close to Joint $\mathrm{A}$ and $\mathrm{B}$, the overall weight that is moved is reduced. To implement the forward kinematics, $\mathrm{q}_{1}, \mathrm{l}_{1}$ and $\mathrm{l}_{2}$ are known. Thus, $\mathrm{x}_{\mathrm{C}}, \mathrm{y}_{\mathrm{C}}, \theta$ and $\mathrm{q}_{2}$ need to be solved.

In order to help the calculation, it needs to do the auxiliary lines and circles on the free body diagram. Draw a line to link BC. And then draw a circle which radius is CD with Joint $\mathrm{C}$ as the center. Thus, the auxiliary free body diagram was shown below in Fig. 2.

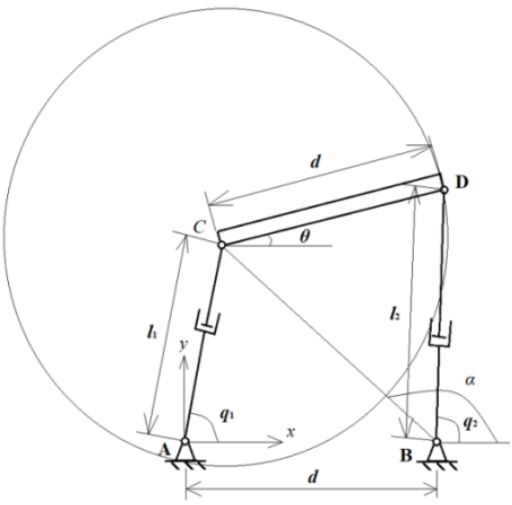

Fig. 2. Auxiliary free body diagram.

Calculate the position of Joint $\mathrm{C}$ :

Calculate the position of Joint D:

$$
x_{C}=l_{1} C_{1}, y_{C}=l_{1} S_{1}
$$

$$
\begin{gathered}
x_{D}=x_{C}+d C_{\theta}=d+l_{2} C_{2} \\
y_{D}=y_{C}+d S_{\theta}=l_{2} S_{2}
\end{gathered}
$$

Use Cosine Law in triangle $\angle \mathrm{DCB}$ to solve for $\angle \mathrm{DCB}$. First, get the length of $\mathrm{BC}$ :

$$
|B C|=\sqrt{\left(x_{C}-d\right)^{2}+y_{C}{ }^{2}}
$$

Write down equation by Cosine Law:

$$
|B C|^{2}+d^{2}-2|B C| d \cos (\angle D C B)=l_{2}{ }^{2}
$$

Expand the above equation:

$$
\left(x_{C}-d\right)^{2}+y_{C}{ }^{2}+d^{2}-2 \sqrt{\left(x_{C}-d\right)^{2}+y_{C}{ }^{2}} d \cos (\angle D C B)=l_{2}{ }^{2}
$$




$$
-2 \sqrt{\left(x_{C}-d\right)^{2}+y_{C}{ }^{2}} d \cos (\angle D C B)=l_{2}^{2}-l_{1}^{2}+2 x_{C} d-2 d^{2}
$$

Simplify the above equation:

$$
\cos (\angle D C B)=-\frac{\frac{l_{2}{ }^{2}-l_{1}{ }^{2}}{2 d}+x_{C}-d}{\sqrt{\left(x_{C}-d\right)^{2}+y_{C}{ }^{2}}}=-\frac{\frac{l_{2}{ }^{2}-l_{1}{ }^{2}}{2 d}+l_{1} C_{1}-d}{\sqrt{l_{1}{ }^{2}-2 l_{1} C_{1} d+d^{2}}}
$$

After got $\angle \mathrm{DCB}$, it can be used to compute $\theta$.

$$
\begin{gathered}
\theta=\angle D C B-(\pi-\alpha)=\angle D C B+\alpha-\pi \\
\text { Where } \sin \alpha=\frac{x_{C}-d}{\sqrt{\left(x_{C}-d\right)^{2}+y_{C}^{2}}}, \cos \alpha=\frac{y_{C}}{\sqrt{\left(x_{C}-d\right)^{2}+y_{C}{ }^{2}}}
\end{gathered}
$$

There are two solutions for $\alpha+\theta$, which are the intersection points between Circle $C$ and Circle B (B as the center and BD as radius). Also, both of two solutions corresponding to one $\theta$. Since in the real life, the stretcher cannot upside down, thus only the positive solution will be taken.

To make the solution for forward kinematics exist, equation (16) has to be valid.

$$
|B C|-d<l_{2}<|B C|+d
$$

\subsection{Dynamics}

Before analyzing the dynamics of this mechanism, some simplification needs to be made. Assume center of mass of CD is at Joint C, COM of AC is at A and COM of BD is at B, so that the translation of link $\mathrm{AC}$ and $\mathrm{BD}$ will not produce energy and also we can ignore energy generated by rotation because $\dot{\theta}$ is too complicated to calculate.

Take line $\mathrm{AB}$ as zero potential energy plane:

$$
\begin{aligned}
& x_{C}=l_{1} C_{1}, y_{C}=l_{1} S_{1} \\
& \dot{x_{C}}=\dot{l_{1}} C_{1}-l_{1} S_{1} \dot{q_{1}}, \dot{y_{C}}=\dot{l_{1}} S_{1}+l_{1} C_{1} \dot{q_{1}}
\end{aligned}
$$

Calculate the potential energy:

$$
P=m l_{1} g S_{1}
$$

Calculate the kinematic energy:

$$
K=0.5 m{\dot{x_{C}}}^{2}+0.5 m \dot{y_{C}}{ }^{2}=0.5 m\left({\dot{l_{1}}}^{2}+{l_{1}}^{2}{\dot{q_{1}}}^{2}\right)
$$

Calculate the total system energy:

$$
L=K-P=0.5 m\left(\dot{l}_{1}^{2}+l_{1}^{2} \dot{q}_{1}^{2}\right)-m g l_{1} S_{1}
$$

Take the partial derivative of $\dot{q}_{1}$ :

$$
\frac{\partial L}{\partial \dot{q}_{1}}=m l_{1}^{2} \dot{q}_{1}
$$

Take the partial derivative of $\frac{\partial \mathrm{L}}{\partial \dot{q}_{1}}$ :

$$
\frac{d}{d t} \frac{\partial L}{\partial \dot{q}_{1}}=m\left(2 l_{1} \dot{l}_{1} \dot{q}_{1}+l_{1}^{2} \ddot{q}_{1}\right)
$$

Take the partial derivative of $\mathrm{q}_{1}$ :

$$
\frac{\partial \mathrm{L}}{\partial \mathrm{q}_{1}}=-\mathrm{mgl}_{1} \mathrm{C}_{1}
$$

Take the partial derivative of $\mathrm{l}_{1}$ :

$$
\frac{\partial L}{\partial \dot{l}_{1}}=m \dot{l_{1}}
$$

Take the partial derivative of $\frac{\partial \mathrm{L}}{\partial \mathrm{l}_{1}}$ : 


$$
\frac{d}{d t} \frac{\partial L}{\partial \dot{l}_{1}}=m \ddot{l_{1}}
$$

Take the partial derivative of $\mathrm{l}_{1}$ :

$$
\frac{\partial L}{\partial l_{1}}=m l_{1} \dot{q}_{1}{ }^{2}-m g S_{1}
$$

Under the assumption, the energy is not related to actuator $\mathrm{l}_{2}$, so the force calculated at this joint is 0 . Since $\mathrm{l}_{2}$ only influences $\theta$ and it doesn't change $\mathrm{x}_{\mathrm{C}}$ and $\mathrm{y}_{\mathrm{C}}$ at all. In practice, if we change $\theta$ very slowly, the force needed on actuator $l_{2}$ will be close to zero. Thus, it can only calculate the torque and force needed on $\mathrm{q}_{1}$ and $\mathrm{l}_{1}$.

$$
\begin{gathered}
\tau_{1}=\frac{d}{d t} \frac{\partial L}{\partial \dot{q}_{1}}-\frac{\partial L}{\partial q_{1}}=m\left(2 l_{1} \dot{l}_{1} \dot{q}_{1}+l_{1}{ }^{2} \ddot{q}_{1}\right)+m g l_{1} C_{1} \\
F_{1}=\frac{d}{d t} \frac{\partial L}{\partial \dot{l}_{1}}-\frac{\partial L}{\partial l_{1}}=m \ddot{l}_{1}-m l_{1} \dot{q}_{1}^{2}+m g S_{1} \\
F_{2}=0
\end{gathered}
$$

Which can be expressed as:

$$
\boldsymbol{F}=\boldsymbol{A}(\boldsymbol{q}) \ddot{\boldsymbol{q}}+\boldsymbol{C}(\boldsymbol{q}, \dot{\boldsymbol{q}})+\boldsymbol{M}(\boldsymbol{q})
$$

Where

$$
\begin{gathered}
\boldsymbol{F}=\left[\begin{array}{l}
\tau_{1} \\
F_{1} \\
F_{2}
\end{array}\right], \boldsymbol{A}(\boldsymbol{q})=\left[\begin{array}{ccc}
m l_{1}^{2} & 0 & 0 \\
0 & m & 0 \\
0 & 0 & 0
\end{array}\right] \\
\boldsymbol{C}(\boldsymbol{q}, \dot{\boldsymbol{q}})=\left[\begin{array}{c}
2 m l_{1} \dot{l}_{1} \dot{q_{1}} \\
-m l_{1} \dot{q}_{1}^{2} \\
0
\end{array}\right] \\
\boldsymbol{M}(\boldsymbol{q})=\left[\begin{array}{c}
m g l_{1} C_{1} \\
m g S_{1} \\
0
\end{array}\right]
\end{gathered}
$$

Use simple PD control:

Calculate $\mathrm{e}_{1}$ and $\ddot{\mathrm{e}_{1}}$ :

$$
\begin{gathered}
e_{1}=q_{1 d}-q_{1}, e_{2}=l_{1 d}-l_{1}, e_{3}=l_{2 d}-l_{2} \\
\ddot{e}+K_{v 1} \dot{e}+K_{p 1} e=0
\end{gathered}
$$

Calculate $\ddot{q_{1}}$ :

$$
\begin{aligned}
e_{1}=q_{1 d}-q_{1}, e_{2} & =l_{1 d}-l_{1}, e_{3}=l_{2 d}-l_{2} \ddot{\mathrm{q}_{1}} \\
& =-\mathrm{K}_{\mathrm{v} 1} \dot{\mathrm{q}}_{1}+\mathrm{K}_{\mathrm{p} 1}\left(\mathrm{q}_{1 \mathrm{~d}}-\mathrm{q}_{1}\right)
\end{aligned}
$$

Calculate $\ddot{\mathrm{I}_{1}}$ and $\ddot{\mathrm{I}_{2}}$ :

$$
\begin{gathered}
\ddot{l_{1}}=-\mathrm{K}_{\mathrm{v} 2} \mathrm{l}_{1}+\mathrm{K}_{\mathrm{p} 2}\left(\mathrm{l}_{1 \mathrm{~d}}-\mathrm{l}_{1}\right. \\
\ddot{l_{2}}=-K_{v 3} \dot{l_{2}}+K_{p 3}\left(l_{2 d}-l_{2}\right)
\end{gathered}
$$

\section{Animation and analysis}

After a series of calculations, using MATLAB to create animations and generate plots. Then, the results obtained will be analyzed. 


\subsection{MATLAB animation}

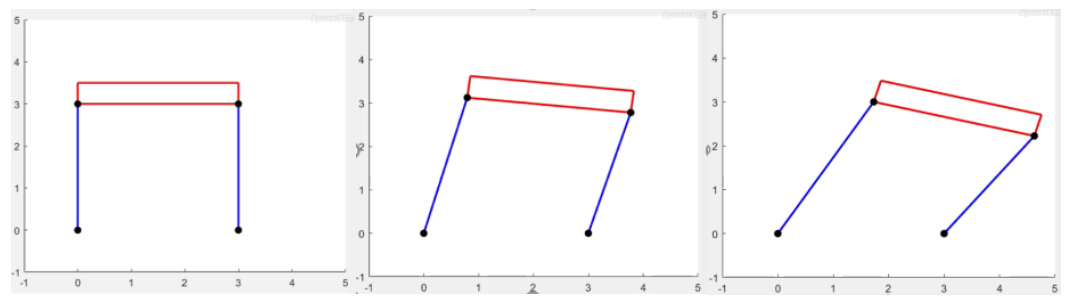

Fig. 3. Auxiliary free body diagram.

Fig. 3 shows the motion trajectory of this mechanism from the original state to the final state when it is working.

\subsection{MATLAB plots}
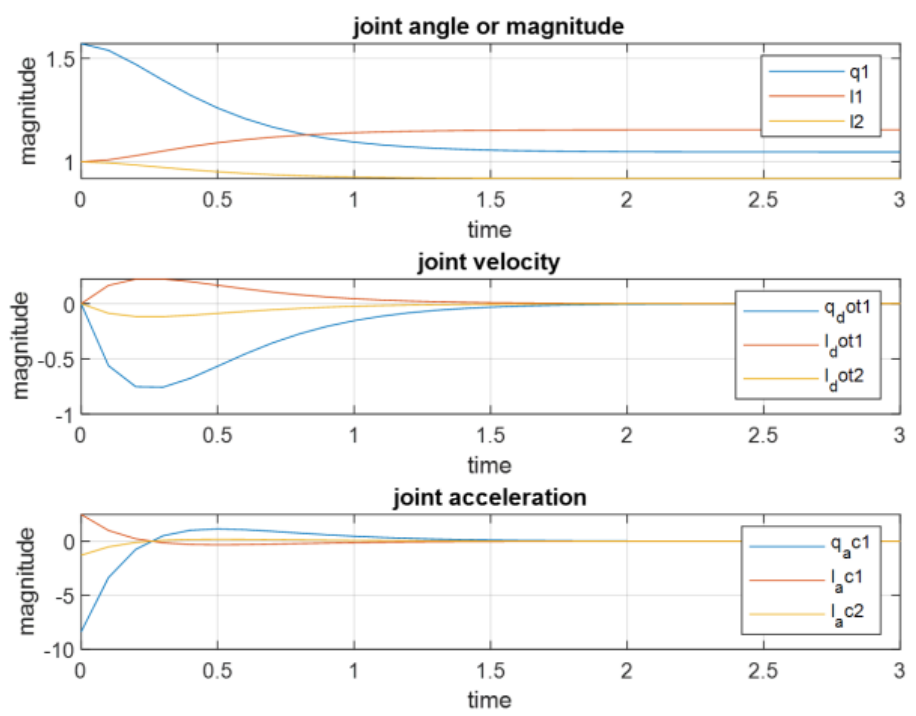

Fig. 4\&5\&6. Joint angle, velocity and acceleration

Fig. $4 \& 5 \& 6$. shows the joint angle, velocity and acceleration of this mechanism from the original state to the final state when it is working. It can be seen that all parameters here are not linear when changing with time. $\mathrm{q}_{1}$ and $\mathrm{l}_{2}$ are consistently drop down with time, but $l_{1}$ increase in the beginning and then decrease until cease. $\dot{q}_{1}$ and $l_{2}$ decrease rapidly in the beginning and then increase slowly until stop. $\dot{l}_{1}$ increase rapidly in the beginning and then decrease slowly until stop. $\ddot{q}_{1}$ and $\ddot{\mathrm{l}_{2}}$ increase quickly at the start and then decrease slowly until shut off. $\ddot{l_{1}}$ increase in the beginning and then decrease until quit. 


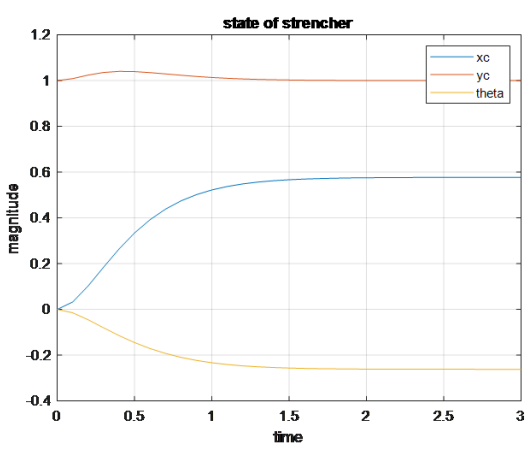

Fig. 7. State of the stretcher

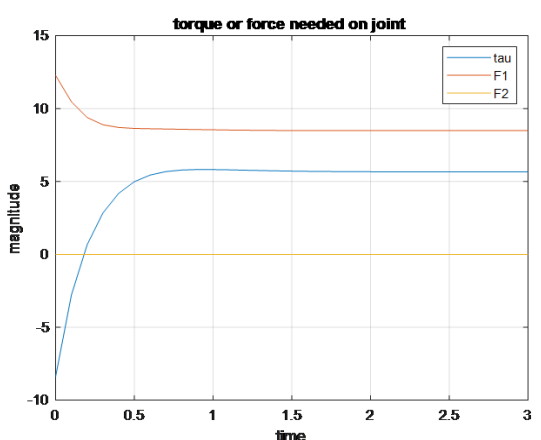

Fig. 8. Torque and force plot

Fig. 7. shows the state change of the stretcher from the original state to the final state when it is in use. This mainly mentions the change in the coordinates of the Joint $\mathrm{C}\left(\mathrm{x}_{\mathrm{C}}\right.$ and $\mathrm{y}_{\mathrm{C}}$ ) and in Rigid Rod CD's angular. As shown in the plot, $\mathrm{y}_{\mathrm{C}}$ increase quickly at the start and then decrease slowly until shut off. $\mathrm{x}_{\mathrm{C}}$ increase quickly at the start and then increase slowly until stop but the change of $\theta$ contrary to $\mathrm{x}_{\mathrm{C}}$. Fig.8. shows the torque and force required of the stretcher. $\tau_{1}$ increase quickly at the start and then increase slowly until stop. Inversely, $F_{1}$ decrease quickly at the start and then decrease slowly until stop. $F_{2}$ always keep 0 .

\subsection{3-D model and animation}

Based on the above analysis, a 3-D model of this mechanism was created by SolidWorks. The prototype of stretcher has four wheels, one base and one bed board. The working processing of this mechanism from the original state to the final state when it is in was shown below in Fig.9.
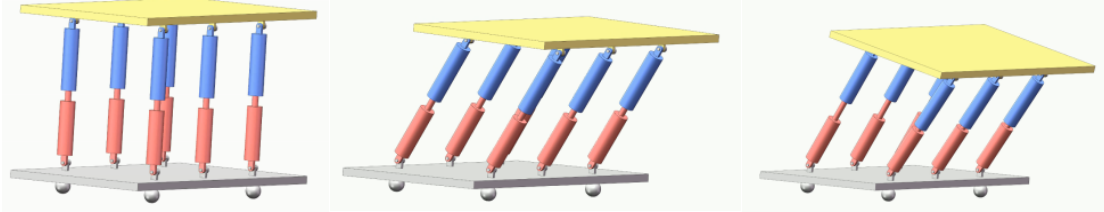

Fig. 9. 3-D Model and animation.

\section{Example simulation}

The rectangular coordinate system is made with the lying human body, the datum is the human heart as the origin of coordinates, and the positions of the human heart and the center of mass of the stretcher coincide. The comfort of the patient was evaluated by the weighted root mean square value method of acceleration in ISO2631-1:1997E Guidelines for Evaluation of Human Body Subjected to Total Body Vibration. The guideline provided the measured accelerometer weight function and weekly weighting coefficient of the lying human body in three directions. In the evaluation criteria, when the weighted root mean square value of acceleration $\mathrm{a}_{\mathrm{v}}$ of the patient's total weighted acceleration was lower than $0.315 \mathrm{~m} / \mathrm{s}^{2}$, the human body felt comfortable; when $a_{v}$ was higher than $0.63 \mathrm{~m} / \mathrm{s}^{2}$, the human body experienced relatively uncomfortable feelings.

In this paper, the influence of the distance between the left and right fulcrum and the center of mass on the comfort of the patient is selected. Suppose the left front fulcrum of 
the stretcher is L1 in the longitudinal direction according to the center of mass, the left front fulcrum of the stretcher is L3 in the transverse direction according to the center of mass, the left rear fulcrum of the stretcher is L2 in the longitudinal direction according to the center of mass, and the right front fulcrum is L4 in the transverse direction according to the center of mass. Now set L1 $=1.05 \mathrm{~m}$ and $\mathrm{L} 2=1.05 \mathrm{~m}$ as fixed values. According to the evaluation criteria, the influence of different distance between left and right fulcrum and center of mass on patient comfort is obtained, as shown in Table 2.

Table 2. Influence of different distance between left and right fulcrum and center of mass on patient comfort.

\begin{tabular}{|l|l|l|l|l|l|l|}
\hline L3 distance & 0.05 & 0.1 & 0.2 & 0.27 & 0.44 & 0.5 \\
\hline L4 distance & 0.5 & 0.45 & 0.35 & 0.27 & 0.1 & 0.05 \\
\hline $\begin{array}{l}\text { Weighted root mean square } \\
\text { value of acceleration( m/ } \\
s^{2} \text { ) }\end{array}$ & 0.3221 & 0.3201 & 0.3184 & 0.3181 & 0.3201 & 0.3221 \\
\hline
\end{tabular}

Set L3 $=0.27$ and L24 $=0.27 \mathrm{~m}$ as fixed values. According to the evaluation criteria, the influence of different distances of fulcrum and center of mass on patient comfort was obtained, as shown in Table 3.

Table 3. Influence of different distances between front and rear fulcrum and center of mass on patient comfort.

\begin{tabular}{|l|l|l|l|l|l|l|}
\hline L1 distance & 0.1. & 0.4 & 0.7 & 1.05 & 1.4 & 1.7 \\
\hline L2 distance & 1.95 & 1.65 & 1.35 & 1 & 0.65 & 0.35 \\
\hline $\begin{array}{l}\text { Weighted root mean square } \\
\text { alue of acceleration( m/ } \\
s^{2} \text { ) }\end{array}$ & 0.3865 & 0.3598 & 0.3375 & 0.3181 & 0.3065 & 0.3037 \\
\hline
\end{tabular}

It can be seen from table 2 and 3 that the farther the distance between the front fulcrum and the center of mass of the stretcher, the closer the distance between the rear fulcrum and the center of mass, the smaller the RMS of weighted acceleration, basically unchanged, and the comfort performance is basically unchanged. The greater the distance between the front fulcrum and the center of mass, the better the comfort of the stretcher.

\section{Conclusion and future extensions}

The team successfully proposed a new mechanism to help transfer patients between beds. The principles and functions of the mechanism was explained in detail. The calculation of kinematics and dynamics (including energy), and PD control in the mechanism was implemented. Also, the motion process was simulated by MATLAB while the plots of angle, torque, and position state were generated. And the prototype and the motion simulation were created by SolidWorks. This design is easy to control with relatively large workspace and it can really avoid the hurt to patients and reduce the burden of nurses in the transferring process. In the future, the team will try to add more degree of freedom in this mechanism and conduct the prototype in clinical trials to get more data to modify the design safer and easier to use.

\section{References}

1. Wang C, Savkin AV, Clout R, Nguyen HT. An Intelligent Robotic Hospital Bed for Safe Transportation of Critical Neurosurgery Patients Along Crowded Hospital Corridors. IEEE Trans Neural Syst Rehabil Eng. 2015 Sep;23(5):744-54. 
2. Agrawal, Sunil \& S. K. 'Statics of In-Parallel Manipulator Systems', Journal of mechanical design. vol. 114, no. 4, pp. 564,

3. S. K. Agrawal and A. Fattah, "Reactionless space and ground robots:

4. Novel designs and concept studies," Mechanisms and Machine Theory,

5. vol. 39, pp. 25-40, 2004.

6. K Pathak, J Franch, SK Agrawal, "Velocity and position control of a wheeled inverted pendulum by partial feedback linearization", IEEE Transactions on robotics 21 (3), 505-513, 2005.

7. H Zhang, SK Agrawal, "Kinematic Design of a Dynamic Brace for Measurement of Head/Neck Motion”, IEEE Robotics and Automation Letters 2 (3), 1428-1435, 2017.

8. L. Ren, X. Zhang, J. Wang, S. Tang and N. Gong, "Design of hospital beds center management information system based on HIS," 2017 IEEE International Conference on Bioinformatics and Biomedicine (BIBM), Kansas City, MO, 2017, pp. 1093-1096.

9. SK Banala, SH Kim, SK Agrawal, JP Scholz, "Robot assisted gait training with active leg exoskeleton (ALEX)", IEEE Transactions on Neural Systems and Rehabilitation Engineering 17 (1), 2-8, 200

10. A. Kassem, M. Hamad, C. E. Moucary, E. Nawfal and A. Aoun, "MedBed: Smart medical bed," 2017 Fourth International Conference on Advances in Biomedical Engineering (ICABME), Beirut, 2017, pp. 1-4. 